\title{
A nnouncements
}

\section{Calendar of Forthcoming Meetings}

2000

Mar. $\quad 17-20$ (Nara) International Symposium on Chaos and Order in Chemistry (ISCOC2000).

Contact: Prof. Takeko Mastumura, Chairperson, Nara University of Education, Takabatake-cho, Nara, 630-8528, Japan (e-mail:takeko@nara-edu.ac.jp).

$18-20$ (Kyoto) International Symposium on Prospects of Bioelectrochemistry in the $21 \mathrm{st}$ Century (PBE21).

Contact: The Secretariat of PBE21, Assoc. Prof. Kenji Kano, Division of Applied Life Sciences, Graduate School of Agriculture, Kyoto University, Kyoto 606-8502, Japan (e-mail: kkano@kais.kyoto-u.ac.jp).

May $8-10($ Warsaw $)$

VI International Symposium CHEMISTRY FORUM 2000.

Contact: Dr. Iwona Głuch, Faculty of Chemistry, Warsaw University of Technology, ul. Noakowskiego 3, 00-664 Warsaw, Poland (e-mail: forum@ch.pw.edu.pl; Website http://www.ch.pw.edu.pl/forum/index.html).

$15-17$ (Montpellier) IXth International Symposium on Luminescence.

Spectrometry in Biomedical and Environmental Aanalysis - Spectroscopic and Imaging Detection Techniques.

Contact: Prof. Dr. Dan A. Lerner, Chairman, University of Montpellier, Ecole Nationale Supérieure de Chimie, 8, Rue de I'Ecole Normale, F-34296 Montpellier cedex 5, France (e-mail: lerner@enscm.fr).

$17-18$ (Nagaoka)

June $\quad 25-29$ (Kyoto)

\section{The 61st Symposium on Analytical Chemistry.*}

XI ICPPP: XI International Conference on Photothermal and Photoacoustic Phenomena.

Contact: Dr. Kenji Katayama, the Secretariat of XI ICPPP, Department of Advanced Materials Sciences, Graduate School of Frontier Sciences, University of Tokyo, 7-3-1, Hongo, Bunkyo-ku, Tokyo 113-8656, Japan (e-mail: icppp@laser.t.u-tokyo.ac.jp; Website http://laser.chem.t.u-tokyo.ac.jp/icppp/).

$25-29$ (Warsaw) 8th International Conference on Flow Analysis.

Contact: Prof. Marek Trojanowicz, Conference Chaimran, Department of Chemistry, University of Warsaw, Pasteura 1, 02-093 Warsaw, Poland (e-mail: trojan@chem.uw.edu.pl; Website http://www. congress.pbp.pl/flow/)

$25-30$ (Seattle) HPLC '2000: 24th International Symposium \& Exhibit on High Performance Liquid Phase Separations \& Related Techniques.

Contact: Janet Cunningham, Barr Enterprises, P. O. Box 279, Walkersville, MD 21793 USA (e-mail: Janetbarr@aol.com)

Sep. $\quad 3-9$ (Lisboa)

Contact: Dr. Christina Oliveira, Departamento de Química e Bioquímica, Faculdade de Ciências Universidade de Lisboa, Edifício C1-Piso 5, P-1700

\section{ICAS 2001}

IUPAC International Congress on Analytical Sciences 2001 6 (Mon.) - 10 (Fri.) Aug. 2001

@Waseda University, Tokyo, Japan

Chairman: Prof. T. SAWADA (The Univ. of Tokyo)

Vice-Chairman: Prof. H. NAKAMURA (Science Univ. of Tokyo)

Prof. Y. UMEZAWA (The Univ. of Tokyo)

Prof. K. MATSUMOTO (Waseda University)

http://wwwsoc.nacsis.ac.jp/jsac/icas2001

e-mail: icas2001@ @laser.t.u-tokyo.ac.jp 
Lisboa (e-mail: euroanalysisxi@fc.ul.pt; Website http://elixir.cc.fc.ulpt/ euroanalysisxi).

$\begin{array}{ll} & 26-28 \text { (Okayama) } \\ \text { Dec. } & 18-20 \text { (Tirupati) }\end{array}$

The 49th Annual Meeting of the Japan Society for Analytical Chemistry.*

ISAME-2. International Seminar on Analytical Techniques in Monitoring the Environment.

Contact: Prof. S. Jayarama Reddy, Director 2nd ISAME, Department of

Chemistry, Sri Venkateswara University, Tirupati-517 502, India (e-mail: jreddy_s

@ yahoo.com).

*Contact: The Japan Society for Analytical Chemistry, 26-2, Nishigotanda 1-chome, Shinagawa-ku, Tokyo 141-0031, Japan.

\section{BUNSEKI KAGAKU}

Vol. 49 No.1 January, 2000

Foreword

Toshiyuki Hobo 1

\section{Research Papers}

Computer-Assisted Identification of Additives Included in Polymers by Combining MS, 'H-NMR, ${ }^{31}$ P-NMR and LC/MS

Keiji Saito and Toshio Ogawa 3

Collection of Laser-Sampled Particles in

Solutions Followed by ICP-MS for the Analysis

of Sintered Silicon Nitride

Tomokazu Tanaka, Jun Kuramata, Mikio Seki and Masataka

Hiraide 11

Determination of Azide Ion by Ion

Chromatography with a Graphitized Carbon

Column

Akinori Isozaki, Hiroto Shimamura, Yukitoki Morita,

Hisomu Nagashima and Tadao Okutani 15

$X$-ray Fluorescence Spectrometry Using the

JRRM800 Reference Materials Series and Its

Application to the Determination of Spinel Raw

Material Components

Hideo Asakura, Katsushige Ikegami and Hisanobu Wakita 21

Synthesis of Polymer Particles with Specific

Binding Sites for Lysozyme by a Molecular

Imprinting Technique and Its Application to a

Quartz Crystal Microbalance Sensor

Kazuko Hirayama and Kei Kameoka 29

Technical Papers

Report on Interlaboratory Testing for the

Determination of Nitrite and Nitrate in River-

Water Samples by Flow-Injection Analysis

Keiro Higuchi, Ryozo Goto, Takuji Kawashima, Koichi

Oguma, Akira Kawase and Hisako Ogura 35
Rapid Determination of Arsenic in Thermally

Cracked Gasoline by Graphite-Furnace AAS

Yoshikazu Nakamoto 43

Online Measurement of Organic Chlorides Using

an Atmospheric-Pressure Chemical Ionization

Ion-Trap Mass Spectrometer

Yuichiro Hashimoto, Masuyoshi Yamada, Masao Suga,

Kouichi Kimura, Minoru Sakairi, Shinji Tanaka, Mamoru

Mizumoto and Masami Sakamoto 49

Spectrophotometric Determination of Cyanide

with Isonicotinic Acid-Sodium Barbiturate

Eiko Nakamura and Michiko Yagi 55

Notes

Sequential Determination of Chromium(VI) and

Chromium(III) by Potentiometric Flow-Injection

Method

Hiroki Ohura, Toshihiko Imato, Ikuo Matsuo and Sumio

Yamasaki 59

Fluorometric Determination of Asulam in Water

by Using Solid-Phase Extraction

Motoshi Nakamura, Tomoko Yasukawa, Tetsuya Igarashi,

Shinkichi Yamada and Sen-ichi Aizawa 65

Digest of Doctoral Dissertation

Development of Determination Methods for Ultra-

Trace Metal Ions of Medical and Environmental

Importance by HPLC

Makoto Sato 69

Development of Simple Methods for the Visual

Determination of Trace Metal Ions by Using

Characteristic Color-Forming Reaction Systems

of Metal Chelates

Akihiko Ishida 71 